\title{
Un médecin qui a fait beaucoup de choses différentes
}

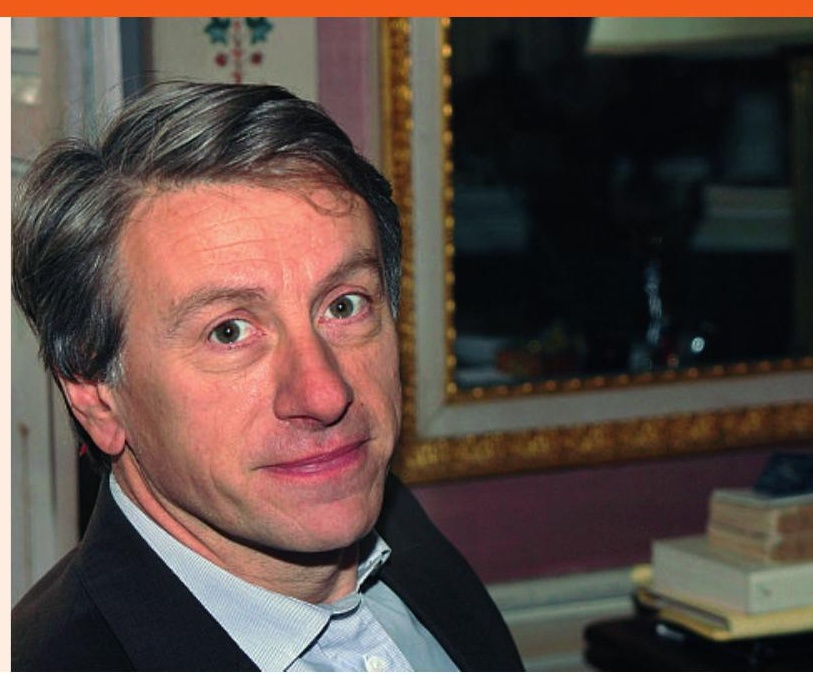

Jean Martin

jean.martin@saez.ch
On connaît Jean-Christophe Rufin pour son engagement au sein de Médecins sans frontières et d'Action contre la faim notamment, et par ses livres. Carrière marquée par des allers et retours entre médecine clinique et action humanitaire, des rebondissements, le passage par Sciences Po et un cabinet ministériel, entre autres choses ... au cours de laquelle il a rassemblé de multiples matériaux utilisés (aussi) dans une importante activité d'écriture: sur l'action humanitaire et ses corollaires socio-politiques puis dans des romans inspirés par ses pérégrinations - qui ont connu le succès (L'Abyssin, Rouge Brésil qui a obtenu le Prix Goncourt 2001, en particulier). Aujourd'hui ambassadeur de France au Sénégal, élu en 2008 à l'Académie française, il vient d'écrire ce qu'il sous-titre "Chroniques d'un médecin nomade». Extraits et commentaires sur quelques sujets d'intérêt pour qui, comme celui qui rédige ces lignes, s'est beaucoup intéressé à la médecine et ses rapports avec la santé publique, à la coopération et à l'aide humanitaire.

\section{De la hiérarchie hospitalière française à I'action de terrain}

Petit-fils d'un praticien du Berry chez lequel il a vécu ses dix premières années en développant une admiration profonde pour la médecine, Rufin fait ses études à Paris, où il passe l'épreuve de l'internat et travaille en milieu hospitalier. Attiré par l'action humanitaire, il est amené une première fois à demander un congé, pour une mission à Djibouti, et se heurte au peu de considération de son chef pour un tel travail: "C'est des vacances que vous voulez, hein?». Plus loin: «Le professeur appartenait à cette génération de grands patrons pour qui la souffrance n'apparaissait intéressante que lorsqu'elle était individuelle. Il n'avait pas encore été contaminé par les approches collectives de la médecine de catastrophe. Face à une foule souffrante, il cessait d'être un médecin et redevenait simplement un grand bourgeois.» Sensibiliser à la dimension de santé publique et communautaire était difficile à l'époque (vers 1980); en réalité, cela le reste aujourd'hui.

Après ses premiers engagements: «J'étais revenu d'Afrique avec une cassure qui répartissait la médecine en deux ordres: d'un côté était l'outil, la méde- cine comme équivalent de la mécanique automobile (...) de l'autre ce que j'avais jusque-là appelé médecine était, au-delà des maladies et des organes, un appel vers l'humain, un intérêt avide pour le siècle, la diversité du monde, les constructions et les destructions produites par l'homme.» Son intérêt pour le domaine se confirme, parce qu'on est à l'interface de la médecine, des sociétés avec leurs différences culturelles et matérielles, et de la politique. «J'y suis entré parce que l'engagement humanitaire que je découvrais révélait qu'il n'était pas hors du monde, comme l'hôpital. Il était plutôt au cœur du monde, de ses luttes, de sa violence. La politique était à la fois son sujet et son quotidien.»

\section{Humanitaire, guerres civiles, place dans I'Histoire ...}

Sur la difficulté à parvenir à ceux qui sont en grandes difficultés: «Partout, les victimes, les laissés-pourcompte, sont cachés, difficiles à atteindre. Partout des forces politiques font écran entre eux et ceux qui viennent leur porter secours (...) Pour arriver jusqu'à ceux qui ont besoin d'aide, il faut d'abord comprendre où ils se trouvent, qui les opprime, qui les représente et pour servir quels intérêts.» Paraphrasant Clausewitz, Rufin a cette formule: «L'humanitaire est la poursuite de la diplomatie par d'autres moyens que la guerre.»

$\mathrm{Au}$ début des années 1990, il entre au cabinet du ministre de la Défense pour s'occuper d'aspects humanitaires, dans le cadre en particulier d'opérations de maintien de la paix. Il est impliqué dans plusieurs missions délicates en ex-Yougoslavie. A propos de la guerre de Bosnie: «Guerre de voisins, guerre entre gens profondément semblables, j'entrais dans le monde abominable et ridicule de cette tragédie balkanique. Elle faisait écho en chacun de nous car elle procédait d'un sentiment que nous avons tous éprouvé un jour ou l'autre: la haine du prochain, forme à peine transposée de la si banale haine de soi.» Il cite cette terrible formule de Marcel Achard: «Ce qu'il y a de bien avec les guerres civiles, c'est qu'on peut rentrer dîner à la maison.»

«Quotidiennement, dans l'humanitaire, on constate la difficulté de nommer les situations historiques. Ce qui se déroule devant nous, les convulsions 


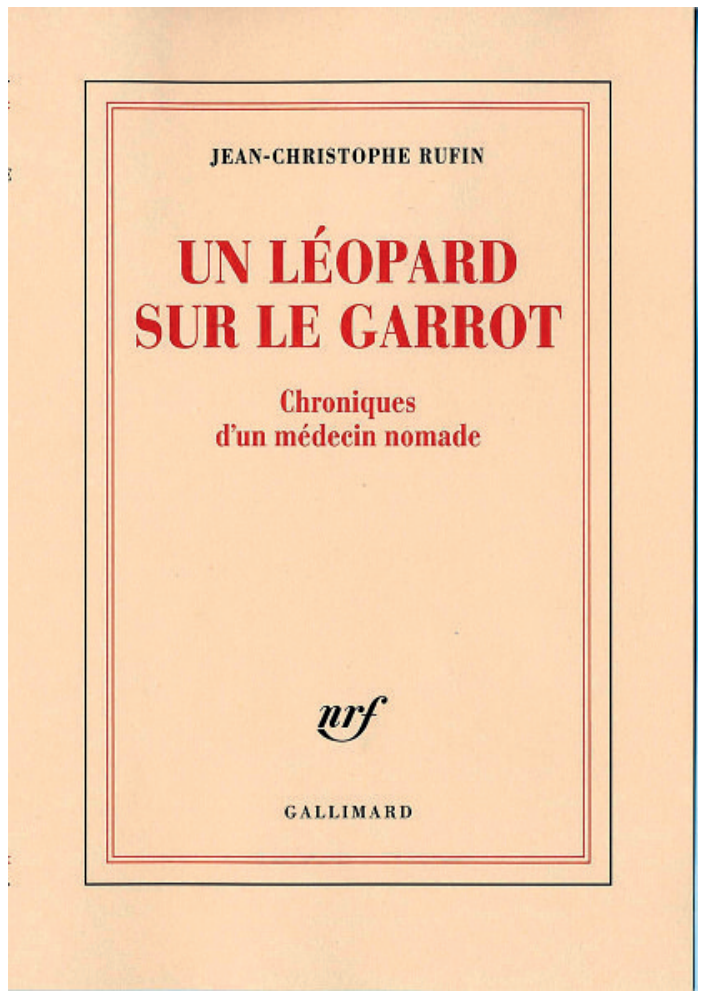

Rufin JC. Un léopard sur le garrot - Chroniques d'un médecin nomade. Paris: Gallimard; 2008.

des peuples, ces évènements qui par la suite portent un nom et le conservent dans l'Histoire, se présentent à l'observateur direct sous des formes partielles et déroutantes. Bien après et parce qu'on le lui dira, il comprendra qu'il a vécu une immense bataille, une date majeure.» Remarque qui fait écho à ce que, à propos de révolutions scientifiques, a dit l'épistémologue Thomas Kuhn [1]; qu'on ne le réalise guère quand on est en plein dans une telle révolution, que ce n'est qu'après qu'on prend conscience qu'il s'agissait d'un moment historique.

\section{Médecine technique et médecine qui privilégie l'écoute, qui respecte}

Ayant débuté sa carrière par une formation médicohospitalière classique (en neurologie), l'auteur est revenu à plusieurs reprises à la clinique. Eclairages: Un de mes patrons «avait coutume de dire qu'il est plus nécessaire (et plus difficile) d'intéresser un auditoire à un SDF (sans domicile fixe) entré pour une banale cuite qu'à une forme rare de lupus ou de maladie de Horton». Plus loin, il fait référence à la médecine «qui se fait avec les yeux, les mains, les oreilles et beaucoup d'intuition, car les SDF s'expriment peu et mal sur leur corps. La plupart de leurs plaintes ne correspondent à rien de sérieux, tandis que les vraies maladies, celles qui les rongent et les tuent, sont chez eux particulièrement muettes.»

Rufin aborde aussi le thème de la mort, que la médecine triomphante du troisième tiers du $\mathrm{XX}^{\mathrm{e}}$ siècle n'admet plus, considère toujours comme une défaite, en se laissant tenter par l'acharnement. Il lui paraît qu'elle n'est plus respectée comme le faisaient la médecine et la société d'avant: «Ce respect de la mort étendait aussi sa protection sur tous ceux qui, par leur grand âge, l'intensité de leur souffrance ou la gravité de leur pathologie, étaient condamnés. La sagesse médicale consistait à savoir jusqu'où il était nécessaire de se battre. Au-delà d'un certain seuil il fallait respecter la paix du patient et le laisser accueillir la mort avec sérénité (...) Malheureusement, nous avons goûté à la toute-puissance.»

En guise de résumé: après trente cinq ans de médecine et de beaucoup d'autres choses, écrivain reconnu et académicien, ambassadeur, Rufin écrit: «La médecine est ma vie, toute la vie. Aujourd'hui que je lui parais si peu fidèle, $j$ 'en suis plus proche que jamais. J'ai envie de raconter cela, de montrer cette unité.»

\section{Référence}

1 Kuhn TS. The Structure of Scientific Revolutions. Chicago: University of Chicago Press; 1962. 